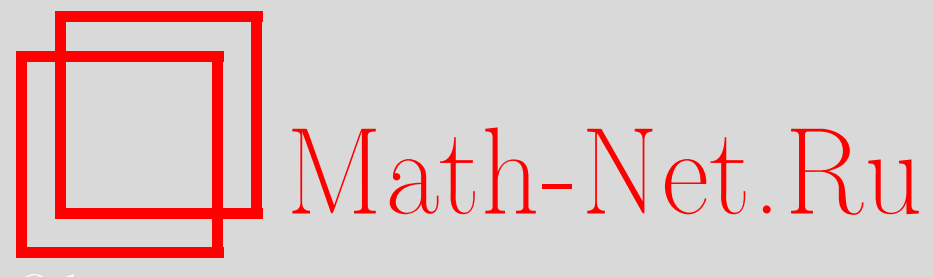

Д. В. Беломестный, Восстановление генерального распределения по распределению некоторых статистик, Теория вероятн. и ее примен., 2004, том 49, выпуск 1, 3-20

DOI: https://doi.org/10.4213/tvp233

Использование Общероссийского математического портала MathNet.Ru подразумевает, что вы прочитали и согласны с пользовательским соглашением

http://www . mathnet.ru/rus/agreement

Параметры загрузки:

IP : 54.198 .67 .100

26 апреля 2023 г., 14:49:05

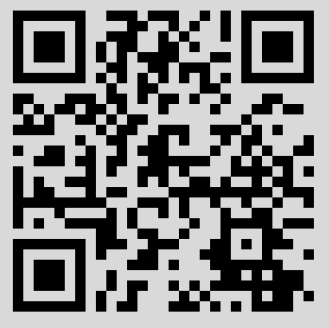




\section{ВОССТАНОВЛЕНИЕ ГЕНЕРАЛЬНОГО РАСПРЕДЕЛЕНИЯ ПО РАСПРЕДЕЛЕНИЮ НЕКОТОРЫХ СТАТИСТИК ${ }^{1)}$}

Исследуется проблема характеризации распределения независимых одинаково распределенных случайных величин $X_{1}, \ldots, X_{m}$ (генеральное распределение) по распределению линейной статистики и статистики максимума с положительными коэффициентами. Получены необходимые и достаточные условия, при которых такая характеризация имеет место.

Ключевые слова и фразы: характеризация распределений по распределению статистик, линейная статистика, статистика максимума.

Введение. При решении статистических задач проверки гипотез о виде распределения часто возникает ситуация, когда мы имеем доступ не к исходной выборке $X_{1}, \ldots, X_{m}$ (генеральное распределение), а к некоторым образом преобразованной. Наиболее часто такими преобразованиями являются линейное преобразование, суперпозиция элементов выборки и выделение максимального элемента. Другими словами, мы приходим к необходимости делать статистические выводы о распределении исходной выборки (генеральном распределении) на основе некоторых статистик от нее. Основными такими статистиками, соответствующими вышеуказанным преобразованиям, являются линейная статистика

$$
L=\beta_{1} X_{1}+\cdots+\beta_{m} X_{m}
$$

и статистика максимума

$$
M=\max \left(\frac{X_{1}}{\beta_{1}}, \ldots, \frac{X_{m}}{\beta_{m}}\right),
$$

где $\beta_{k}$ - положительные действительные числа.

Независимо от характера решаемой статистической задачи (оценивание, проверка гипотез), основополагающим является вопрос о единственности восстановления исходного распределения по распределению

* Московский государственный университет им. М. В. Ломоносова, Ленинские горы, 119992 Москва, Россия; e-mail: denbelom@mail.ru

1) Работа выполнена при частичной поддержке РФФИ (проект № 00-01-00661). 
статистик $L$ и $M$. Работа посвящена получению условий, при которых распределение статистик $L$ и $M$ характеризует генеральное распределение. Что касается вопроса восстановления генерального распределения по распределению линейной статистики, то для частного случая, когда все коэффициенты $\beta_{k}$ равны 1 , эта задача рассматривалась в работах [1] и [8], где получены некоторые достаточные условия однозначности такого восстановления. Эти условия связаны либо с единственностью продолжения соответствующей характеристической функции с любого интервала, содержащего начало координат, либо с ее необращением в нуль (что эквивалентно непрерывности аргумента). Единственным результатом в общем случае является указание Линника (см. [5]) на то, что в классе распределений, имеющих все моменты и удовлетворяющих условиям единственности решения проблемы моментов, распределение статистики $L$ однозначно определяет генеральное распределение. Проблема характеризации генерального распределения распределением статистики $M$, по сути, нигде ранее не рассматривалась, если не считать случая равенства всех $\beta_{k}$ единице (см. [3]), в котором она становится тривиальной.

1. Формулировка основных результатов. Перепишем нашу линейную статистику в виде

$$
L=b_{1}\left(X_{11}+\cdots+X_{1 k_{1}}\right)+\cdots+b_{n}\left(X_{n 1}+\cdots+X_{n k_{n}}\right)
$$

где $k_{i}$ - натуральные числа и $b_{i}$ удовлетворяют условию

$$
b_{1}<\cdots<b_{n}
$$

и введем при $n>1$ следующие обозначения:

$$
\begin{gathered}
q_{i}=\frac{k_{i}}{k_{n}}, \quad h_{i}=\frac{b_{i}}{b_{n}}, \quad i=1, \ldots, n-1, \\
\tau(z)=1+q_{1} h_{1}^{z}+\cdots+q_{n-1} h_{n-1}^{z} .
\end{gathered}
$$

Теорема 1.1. Пусть две Функиии распределения $F(x)$ u $G(x)$ таковь, что $L_{F} \cong L_{G}$. Положим $\alpha=\max \{\operatorname{Re} z: \tau(z)=0\}$. Eсли выполнено одно из следующих условий:

1) $n=1$,

2) $n>1, q_{1}+\cdots+q_{n-1} \leqslant 1$,

3) $n>1, q_{1}+\cdots+q_{n-1}>1$ и $F$ обладает моментом порядка $\alpha$, mо $f(t)=g(t)$ nрu $|t|<\delta$, где $f(t)$ u $g(t)$ - характеристические функиии, соответствующие $F(x)$ u $G(x), u \delta=\min \{t>0:|f(t)|=0\}$. Eсли же условия 1), 2) и 3) нарушень, то существуют такие две функиии распределения $F_{0}(x)$ и $G_{0}(x)$, обладающие моментами порядка $\alpha-\varepsilon$ 
для любого $\delta>\varepsilon>0$ и порождающие одинаковое распределение статистики L, что соответствующие характеристические функиии не равны тождественно ни на одном интервале, содержащем точку 0.

Следствие 1.1. Пусть в условиях теоремы $1.1 f(t)$ такова, что для нее проблема продолжения с любого интервала, содержащего 0 , имеет единственное решение (см. [2]). Тогда $F \equiv G$.

Следствие 1.2. Пусть в условиях теоремь $1.1 f(t)$ не обращается в нуль на $(-\infty, \infty)$. Тогда $F \equiv G$.

Назовем полным типом распределения множество всех функций распределения вида $G((x-a) / b)$, где $G(x)$ - функция распределения, $a \in \mathbf{R}$ и $b>0$.

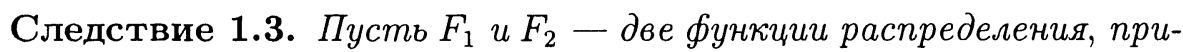
надлежащие некоторому общему полному типу, и $F=F_{1} * F_{2}$. Если характеристическая функиия $F(x)$ не обращается в нуль на всей прямой, то тип $F_{1}$ и $F_{2}$ однозначно определяется по $F$.

Обратимся теперь к статистике $M$. Перепишем ее в виде

$$
M=\max \left(\frac{X_{11}}{b_{1}}, \ldots, \frac{X_{1 k_{1}}}{b_{1}}, \ldots, \frac{X_{n 1}}{b_{n}}, \ldots, \frac{X_{n k_{n}}}{b_{n}}\right),
$$

где $k_{i}$ - натуральные числа и $b_{i}$ удовлетворяют условию $b_{1}<\cdots<b_{n}$, и введем при $n>1$ следующие обозначения:

$$
\begin{gathered}
q_{i}=\frac{k_{i}}{k_{1}}, \quad h_{i}=\frac{b_{i}}{b_{1}}, \quad i=2, \ldots, n, \\
\tau_{1}(z)=1+q_{2} h_{2}^{-z}+\cdots+q_{n} h_{n}^{-z} .
\end{gathered}
$$

Теорема 1.2. Пусть две функции распределения $F(x)$ и $G(x) m a-$ ковы, ито $M_{F} \cong M_{G}$. Положим $\alpha_{1}=\max \left\{\operatorname{Re} z: \tau_{1}(z)=0\right\}$. Если выполнено одно из следующих условий:

1) $n=1$,

2) $n>1, q_{2}+\cdots+q_{n} \leqslant 1$,

3) $n>1, q_{2}+\cdots+q_{n}>1$ и F обладает моментом порядка $\alpha_{1}$, то $F(x)=G(x)$ при $x>0$. Если же условия 1), 2) и 3) нарушень, то существуют две различные функиии распределения $F_{0}(x)$ и $G_{0}(x)$, сосредоточеннье на $(0, \infty)$, обладаюшие моментами порядка $\alpha_{1}-\delta$ для любого $\alpha_{1}>\delta>0$ и порождающие одинаковое распределение статистики $M$.

Следствие 1.4. Пусть в условиях теоремы $1.2 F(0)=0$. Тогда $F(x)=G(x)$ при $x \in \mathbf{R}$. 


\section{2. Вспомогательные результаты.}

Лемма 2.1. Пусть $X_{1}, \ldots, X_{n}$ - независимые одинаково распределенные случайные величины и

$$
L=a_{1} X_{1}+\cdots+a_{n} X_{n}
$$

где $a_{1}, \ldots, a_{n}-$ конечные действительные числа. Тогда $\mathbf{E}|L|^{r}<\infty$ эквивалентно $\mathbf{E}\left|X_{1}\right|^{r}<\infty$ для любого $r>0$.

Д о к азат е льст в о. Докажем вначале импликацию $\mathbf{E}|L|^{r}<$ $\infty \Rightarrow \mathbf{E}\left|X_{1}\right|^{r}<\infty$. Без ограничения общности рассуждений будем считать, что $a_{1} \neq 0$. Положим $b=-m\left(a_{2} X_{2}+\cdots+a_{n} X_{n}\right)$, тогда

$$
\left|a_{1} X_{1}-b\right| \leqslant \max _{1 \leqslant k \leqslant n}\left|S_{k}-m\left(S_{k}-S_{n}\right)\right|
$$

где $S_{k}=a_{1} X_{1}+\cdots+a_{k} X_{k}, k=1, \ldots, n$. Согласно неравенству Леви (см. $[7$, c. 78$]$ ),

$$
\mathbf{P}\left\{\left|a_{1} X_{1}-b\right| \geqslant x\right\} \leqslant \mathbf{P}\left\{\max _{1 \leqslant k \leqslant n}\left|S_{k}-m\left(S_{k}-S_{n}\right)\right|\right\} \leqslant 2 \mathbf{P}\left\{\left|S_{n}\right| \geqslant x\right\},
$$

что влечет $\mathbf{E}\left|a_{1} X_{1}-b\right|^{r} \leqslant 2 \mathbf{E}\left|S_{n}\right|^{r}=2 \mathbf{E}|L|^{r}$. Далее,

$$
\begin{array}{lll}
\text { при } & r \leqslant 1 & \mathbf{E}\left|a_{1} X_{1}\right|^{r} \leqslant \mathbf{E}\left|a_{1} X_{1}-b\right|^{r}+|b|^{r}<\infty, \\
\text { при } & r>1 & \mathbf{E}\left|a_{1} X_{1}\right|^{r} \leqslant 2^{r-1} \mathbf{E}\left|a_{1} X_{1}-b\right|^{r}+2^{r-1}|b|^{r}<\infty .
\end{array}
$$

Для доказательства утверждения в обратную сторону достаточно заметить, что

$$
\begin{array}{lll}
\mathbf{E}|L|^{r} \leqslant\left|a_{1}\right|^{r} \mathbf{E}\left|X_{1}\right|^{r}+\cdots+\left|a_{n}\right|^{r} \mathbf{E}\left|X_{n}\right|^{r} & \text { при } & r \leqslant 1, \\
\mathbf{E}|L|^{r} \leqslant n^{r}\left(\left|a_{1}\right|^{r} \mathbf{E}\left|X_{1}\right|^{r}+\cdots+\left|a_{n}\right|^{r} \mathbf{E}\left|X_{n}\right|^{r}\right) & \text { при } & r>1 .
\end{array}
$$

Лемма 2.2. Пусть $X$ и $Y-$ две случайные величины с характеристическими функииями $f(t)$ и $g(t)$. Если $\mathbf{E}|X|^{m}<\infty, \mathbf{E}|Y|^{m}<\infty n p u$ некотором натуральном $m$ и существует стремящаяся $\kappa$ нулю последовательность $t_{n}$ такая, ито

$$
f\left(t_{n}\right)=g\left(t_{n}\right), \quad n \in \mathbf{N},
$$

$m o f^{(l)}(0)=g^{(l)}(0), l=0, \ldots, m$.

Д ок а за т ел ь с т в о. Сразу отметим, что $f(t)$ и $g(t)$ непрерывно дифференцируемы $m$ раз на всей прямой. Так как выполнено (2.1), то, применяя последовательно теорему Ролля к функциям $f^{(l)}(t)-g^{(l)}(t), l=0, \ldots, m$, получим, что сушествует система стремящихся к нулю последовательностей $\left\{t_{l, n}, l=1, \ldots, m, n \in \mathbf{N}\right\}$ таких, что $f^{(l)}\left(t_{l, n}\right)=g^{(l)}\left(t_{l, n}\right), n \in \mathbf{N}$. 
Лемма 2.3. Для любой характеристической функиии $f(t)$ выполнены следуюшие неравенства:

$$
\begin{gathered}
|\operatorname{Im} f(t)| \leqslant \sqrt{2(1-\operatorname{Re} f(t))}, \\
|\operatorname{Im} f(a t)+\operatorname{Im} f(b t)+\operatorname{Im} f(-(a+b) t)| \leqslant(1-\operatorname{Re} f(a t)) \\
+(1-\operatorname{Re} f(b t))+(1-\operatorname{Re} f(-(a+b) t)), \quad a, b \in \mathbf{R} .
\end{gathered}
$$

Д о к а з а т е л ь с т в о. Доказательство первого неравенства имеется в [9], а второго - в [6].

Лемма 2.4. Для того чтобь распределение $F(x)$ с характеристической функиией $f(t)$ имело момент порядка $2 k+\lambda$, гдеп $k \in \mathbf{N}, 0<\lambda<2$, необходимо и достаточно, чтобы для любого конечного $\delta>0$

$$
\int_{0}^{\delta} t^{-\lambda-1}\left(1-\operatorname{Re} \frac{f^{(2 k)}(t)}{f^{(2 k)}(0)}\right) d t<\infty .
$$

Д о к а з а т е л ь с т в о. Доказательство имеется в работе [4].

Теорема 2.1. Пусть функиия $w(x)$ удовлетворяет уравнению

$$
q_{1} w\left(x+\gamma_{1}\right)+\cdots+q_{m} w\left(x+\gamma_{m}\right)+w(x)=0, \quad x>0,
$$

где $q_{i}$ и $\gamma_{i}$ - положительные числа. Обозначим через $\tau(z)$ функиию $1+q_{1} e^{\gamma_{1} z}+\cdots+q_{m} e^{\gamma_{m} z}$ и введем число

$$
\alpha=\min \{\operatorname{Re} z: \tau(z)=0\} .
$$

Тогда если выполнено одно из следующих условий:

1) $q_{1}+\cdots+q_{m}<1$

2) $q_{1}+\cdots+q_{m}=1, \lim _{x \rightarrow \infty}|w(x)|=0$,

3) $\int_{0}^{\infty} e^{-\alpha x}|w(x)| d x<\infty$, то $w(x) \equiv 0$ при $x>0$. При нарушении этих трех условий частными решениями уравнения (2.2) будут функиии вида

$$
e^{\alpha x}\left(c_{1} e^{i \mu_{1} x}+\cdots+c_{l} e^{i \mu_{l} x}\right), \quad x>0,
$$

где числа $\alpha+i \mu_{k}$ суть корни $\tau(z)$ на прямой $\operatorname{Re} z=\alpha<0$ (по нашему предположению множество таких корней не пусто).

Д о к а з а т е л ь с т в о. Если $q_{1}+\cdots+q_{m} \leqslant 1$, то $(2.2)$ после $n$ итераций дает нам

$$
w(x)=(-1)^{n} \sum_{n_{1}+\cdots+n_{m}=n} \frac{n !}{n_{1} ! \cdots n_{m} !} q^{n_{1}} \cdots q_{m}^{n_{m}} w\left(x+n_{1} \gamma_{1}+\cdots+n_{m} \gamma_{m}\right)
$$

откуда

$$
|w(x)| \leqslant\left(q_{1}+\cdots+q_{m}\right)^{n}\left|w\left(x+n \gamma_{*}\right)\right| \longrightarrow 0, \quad n \rightarrow \infty,
$$

где $\gamma_{*}=\min \left\{\gamma_{1}, \ldots, \gamma_{m}\right\}>0$. 
Пусть выполнено условие 2, тогда, применяя к (2.2) преобразование Лапласа, получим

$$
\Phi(z) \tau(z)+M(z)=0
$$

где

$$
\Phi(z)=\int_{0}^{\infty} e^{-z x} w(x) d x, \quad M(z)=\sum_{k=1}^{m} q_{k} e^{\gamma_{k} z} \int_{0}^{\gamma_{k}} e^{-z x} w(x) d x
$$

Лемма 2.5. 1) $\Phi(z)$ аналитична при $\operatorname{Re} z \geqslant \alpha$ и представляется в этой области в виде $\Phi(z)=-M(z) / \tau(z)$.

2) $M(z)$ есть челая функция, ограниченная константой $B=B(c)$ в каждой полуплоскости $\operatorname{Re} z \leqslant c$.

3) Функиия $\tau(z)$ обладает следуюшими свойствами:

(а) $\tau(z)$ является иелой функиией, почти периодической на каждой прямой $\operatorname{Re} z=$ const;

(b) $\tau(z)$ не имеет нулей при $\operatorname{Re} z<\alpha$;

(c) число нулей $\tau(z)$ в любом замкнутом прямоугольнике $\{a \leqslant$ $\operatorname{Re} z \leqslant b, y \leqslant \operatorname{Im} z \leqslant y+1\}$ ограничено числом $N(a, b)$, не зависящим om $y$;

(d) если точка $z_{0}$ удалена от всех нулей $\tau(z)$ на расстояние, большее, чем $\varepsilon>0$, mo $\left|\tau\left(z_{0}\right)\right|>A(\varepsilon)$.

Доказатель с т о. Пункт 1 следует из условия 2) теоремы 2.1 и (2.3), пп. 2 и 3(a) вытекают из определения, а пп. 3(b)-3(d) являются следствием свойств почти периодических функций и доказаны, например, в [6].

Следствие 2.1. Для любого $c>0$ существует такая последовательность $T_{k} \rightarrow \infty$, что $|\tau(z)|>A$ при $|\operatorname{Im} z|=T_{k}, k \in \mathrm{N}, \operatorname{Re} z<c$.

Д ок аз а т е л ь с т в о. Из п. 3(b) следует, что для любого натурального $k$ существуют такое малое $\varepsilon>0$, не зависящее от $k$, и такое число $T_{k} \in[k, k+1]$, что все точки отрезка $\left\{\alpha \leqslant \operatorname{Re} z \leqslant c, \operatorname{Im} z=T_{k}\right\}$ удалены от нулей $\tau(z)$ на расстояние, большее, чем $\varepsilon$. Так как $\tau(z) \neq 0$ при $\operatorname{Re} z<\alpha$, то это же утверждение остается верным для полупрямой Re $z \leqslant c$. Согласно п. $3(\mathrm{~d}),|\tau(z)|>A=A(\varepsilon)$ для всех $z$ из полупрямых $\left\{\operatorname{Re} z \leqslant c, \operatorname{Im} z=T_{k}\right\}$. В силу того, что все нули $\tau(z)$ разбиваются на пары комплексно сопряженных, это же верно и для полупрямых $\{\operatorname{Re} z \leqslant$ $\left.c, \operatorname{Im} z=-T_{k}\right\}$.

По формуле комплексного обращения для преобразования Лапласа имеем для $u>0$

$$
\int_{0}^{u} w(x) d x=\lim _{T \rightarrow \infty} \frac{1}{2 \pi i} \int_{c-i T}^{c+i T} \frac{e^{u z} \Phi(z)}{z} d z
$$


при $c>0$. Функция $-M(z) / \tau(z)$ есть целая функция, равная $\Phi(z)$ при $\operatorname{Re} z>\alpha$. Пусть $h<\alpha<0$, тогда по теореме Коши о вычетах будем иметь

$$
\begin{aligned}
& \frac{1}{2 \pi i} \int_{c-i T}^{c+i T} \frac{e^{u z} M(z)}{z \tau(z)} d z \\
& \quad=\frac{1}{2 \pi i}\left[\left(\int_{h+i T}^{c+i T}-\int_{h-i T}^{c-i T}+\int_{h-i T}^{h+i T}\right) \frac{e^{u z} M(z)}{z \tau(z)} d z\right]+\Phi(0) .
\end{aligned}
$$

Далее, при фиксированном $k$

$$
\left|\int_{h-i T_{k}}^{h+i T_{k}} \frac{e^{u z} M(z)}{z \tau(z)} d z\right| \leqslant \frac{2(k+1) B}{|h| A(|h|-|\alpha|)} e^{h u} \longrightarrow 0, \quad h \rightarrow-\infty,
$$

$\left|\left(\int_{-\infty+i T_{k}}^{c+i T_{k}}-\int_{-\infty-i T_{k}}^{c-i T_{k}}\right) \frac{e^{u z} M(z)}{z \tau(z)} d z\right| \leqslant \frac{B}{A} e^{u c} \frac{1}{\sqrt{c^{2}+T_{k}^{2}}} \longrightarrow 0, \quad k \rightarrow \infty$.

Учитывая, что $\Phi(0)=\int_{0}^{\infty} w(x) d x$, окончательно получим $\int_{u}^{\infty} w(x) d x=0$ для любого $u>0$. Второе утверждение теоремы проверяется непосредственно.

Лемма 2.6. Пусть $0<\mu \leqslant 2, \lambda \geqslant \mu, \beta$ - действительнье числа, тогда существуют такие положительное $A$, неотричательное $D u$ натуральное $m \leqslant 2$, что функция

$$
f(t)=\exp \left[-|t|^{\mu}-A|t|^{\lambda}\left(1+B e^{i(t /|t|) \beta \ln |t|}+D \ln ^{m}|t|\right)\right]
$$

является характеристической для всех $B$ таких, что

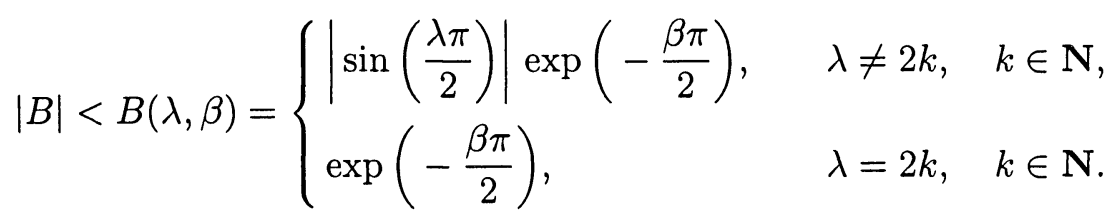

Д о к а з а т л ь с т в о. Пусть сначала $A, B$ и $D$ не превосходят по модулю 1 . В силу интегрируемости и эрмитовости $f(t)$ нам достаточно доказать, что

$$
p(x)=\frac{1}{\pi} \operatorname{Re} \int_{0}^{\infty} e^{-i t x} f(t) d t \geqslant 0, \quad x \in \mathbf{R} .
$$

Рассмотрим в комплексной области $z=t+i s$ прямоугольный контур, образованный пересечением координатных осей и двух прямых $s=-S$ и $t=T$. Так как функция $f(z)$ аналитична в области $\operatorname{Re} z>0, \operatorname{Im} z<0$ и непрерывна на ее границе, то, применяя теорему Коши к этому контуру, мы получим

$$
\begin{aligned}
\int_{0}^{T} e^{-i t x} f(t) d t= & -i \int_{0}^{S} e^{-s x} f(-i s) d s+\int_{0}^{T} e^{-i(t-i S) x} f(t-i S) d t \\
& +i \int_{0}^{S} e^{-i(T-i s) x} f(T-i s) d s
\end{aligned}
$$


Нетрудно показать, что $|f(T-i s)|=O\left(e^{-T^{\mu}}\right)$ при $T \rightarrow \infty$ равномерно по $s$. В силу этого третий интеграл в (2.5) сходится к нулю при $T \rightarrow \infty$, а второй по модулю не превосходит $e^{-S x} \int_{0}^{T}|f(t-i S)| d t=O\left(e^{-S x}\right)$. Обратимся теперь к первому интегралу. Так как

$$
\begin{gathered}
-i \int_{0}^{S} e^{-s x} f(-i s) d s=-i \int_{0}^{\infty} e^{-s x} f(-i s) d s+i \int_{S}^{\infty} e^{-s x} f(-i s) d s \\
\left|\int_{S}^{\infty} e^{-s x} f(-i s) d s\right| \leqslant e^{-S x / 2} \int_{S}^{\infty} e^{-s x / 2}|f(-i s)| d s \leqslant C e^{-S x / 2}
\end{gathered}
$$

то с учетом (2.5) получим

$$
\operatorname{Re} \int_{0}^{\infty} e^{-i t x} f(t) d t=\operatorname{Re}\left(-i \int_{0}^{\infty} e^{-s x} f(-i s) d s\right)+R(x),
$$

где $|R(x)| \leqslant C e^{-S x / 2}$.

Далее, нетрудно доказать существование такого $X$, не зависящего от $\lambda, \mu, A, B$, что при достаточно большом $S, x>X$ и $B$, удовлетворяющем (2.4), правая часть (2.6) будет неотрицательна.

Пусть теперь $x \leqslant X$. Имеем

$$
\begin{aligned}
\int_{0}^{\infty} & e^{-i t x} \exp \left[-t^{\mu}-A t^{\lambda}\left(1+B e^{i \beta \ln t}+D \ln ^{m} t\right)\right] \\
= & \int_{0}^{\infty} \exp \left(-i t x-t^{\mu}\right) d t \\
& +\int_{0}^{T} \exp \left(-i t x-t^{\mu}\right)\left(\exp \left[-A t^{\lambda}\left(1+B e^{i \beta \ln t}+D \ln ^{m} t\right)\right]-1\right) d t \\
& +\int_{T}^{\infty} \exp \left(-i t x-t^{\mu}\right)\left(\exp \left[-A t^{\lambda}\left(1+B e^{i \beta \ln t}+D \ln ^{m} t\right)\right]-1\right) d t \\
& =I_{1}+I_{2}+I_{3} .
\end{aligned}
$$

Используя неравенство $\left|e^{z}-1\right| \leqslant|z| e^{|z|}$, верное для любого комплексного $z$, получим

$$
\begin{aligned}
\left|I_{2}\right| & \leqslant \int_{0}^{T} \exp \left(-t^{\mu}\right) \exp \left(A t^{\lambda}\left(2+\ln ^{2} t\right)\right) A t^{\lambda}\left(2+\ln ^{2} t\right) d t \\
& \leqslant A C_{4} \exp \left(A T^{\lambda}\left(2+\ln ^{2} T\right)\right)
\end{aligned}
$$

и $\left|I_{3}\right| \leqslant C_{5} e^{-T^{\mu / 2}}$. Воспользуемся теперь тем известным фактом, что $\operatorname{Re} I_{1}(x)$ есть функция, положительная на всей оси (см. [6]), и поэтому $\operatorname{Re} I_{1}(x)>\delta>0$ при $x \leqslant X$. Выбирая $T_{0}$ и $A$ подходящим образом, приходим к выводу, что левая часть (2.7) положительна.

Лемма 2.7. Пусть $\alpha>0$ и $\beta$ - действительнье числа, тогда функиия

$$
K(x)= \begin{cases}\exp \left\{-x^{-\alpha}(1+C \cos (\beta \ln x))\right\}, & x>0 \\ 0, & x \leq 0\end{cases}
$$


является функиией распределения при всех $C$ таких, что $|C|<(1+$ $|\beta| / \alpha)^{-1}$.

Д ок а з а т е л с т в о. Проверяется непосредственно.

Лемма 2.8. Функиия распределения $F(x)$ имеет конечный абсолютный момент порядка $\alpha>0$ тогда и только тогда, когда

$$
\int_{-\infty}^{\infty}|x|^{\alpha-1}(1-F(x)+F(-x)) d x<\infty .
$$

Доказательство имеется в [7].

Следствие 2.2. Если $F(0)=0$, то условие

$$
\int_{0}^{\infty} x^{\alpha-1}(1-F(x)) d x<\infty
$$

является необходимьм и достаточньм для существования у $F(x)$ момента порядка $\alpha$.

\section{3. Доказательство основных результатов.}

Д ок а за т ел ь с т в о т е о р е м ы 1.1. Условие $L_{F} \cong L_{G}$ в терминах $f(t)$ и $g(t)$ будет эквивалентно равенству

$$
f^{k_{1}}\left(b_{1} t\right) \cdots f^{k_{n}}\left(b_{n} t\right)=g^{k_{1}}\left(b_{1} t\right) \cdots g^{k_{n}}\left(b_{n} t\right), \quad t \in \mathbf{R} .
$$

Из этого равенства видно, что $g(t) \neq 0$ при $|t|<\delta$, и поэтому можно определить следующие функции:

$$
\zeta(t)=\ln f(t), \quad \xi(t)=\ln g(t), \quad|t|<\delta,
$$

гце мы выбираем непрерывную ветвь логарифмической функции, принимающую вещественные значения при положительных значениях аргумента.

Из (3.1) вытекает, что

$$
k_{1} \zeta\left(b_{1} t\right)+\cdots+k_{n} \zeta\left(b_{n} t\right)=k_{1} \xi\left(b_{1} t\right)+\cdots+k_{n} \xi\left(b_{n} t\right), \quad|t|<b_{n} \delta^{-1} .
$$

Далее, обозначая $\Delta(t)=\zeta(t)-\xi(t)$, мы имеем

$$
k_{1} \Delta\left(b_{1} t\right)+\cdots+k_{n} \Delta\left(b_{n} t\right)=0, \quad|t|<b_{n}^{-1} \delta .
$$

Если $n=1$, то $k_{1} \Delta(t)=0,|t|<\delta$, и утверждение теоремы доказано. Пусть $n>1$, тогда в обозначениях (1.2) уравнение (3.2) перепишется следующим образом:

$$
q_{1} \Delta\left(h_{1} t\right)+\cdots+q_{n-1} \Delta\left(h_{n-1} t\right)+\Delta(t)=0, \quad|t|<\delta .
$$

Определяя $S(u)=\Delta\left(e^{-u}\right)$, получим из $(3.3)$

$$
q_{1} S\left(u+\gamma_{1}\right)+\cdots+q_{n-1} S\left(u+\gamma_{n-1}\right)+S(u)=0, \quad u \in(-\ln \delta, \infty) .
$$


Из теоремы 2.1 следует, что если $q_{1}+\cdots+q_{n-1} \leqslant 1$, то $S(u)=0$ при $u>-\ln \delta$ и поэтому $f(t) \equiv g(t)$ на $(-\delta, \delta)$.

Теперь обратимся к случаю $q_{1}+\cdots+q_{n-1}>1$. Введем снова обозначения

$$
\begin{gathered}
\Delta_{M}(t)=\operatorname{Re} \Delta(t)=\ln |f(t)|-\ln |g(t)|, \\
\Delta_{A}(t)=\operatorname{Im} \Delta(t)=\arg f(t)-\arg g(t) .
\end{gathered}
$$

Уравнение (3.3) теперь распадается на два

$$
\begin{aligned}
q_{1} \Delta_{M}\left(h_{1} t\right)+\cdots+q_{n-1} \Delta_{M}\left(h_{n-1} t\right)+\Delta_{M}(t) & =0, & & |t|<\delta, \\
q_{1} \Delta_{A}\left(h_{1} t\right)+\cdots+q_{n-1} \Delta_{A}\left(h_{n-1} t\right)+\Delta_{A}(t) & =0, & & |t|<\delta .
\end{aligned}
$$

Лемма 3.1. Либо функиия $\Delta_{M}(t)\left(\Delta_{A}(t)\right)$ тождественно равна нулю при $|t|<\delta$, либо существует сходящаяся $\kappa$ нулю последовательность $t_{n}\left(s_{n}\right)$ такая, что

$$
\Delta_{M}\left(t_{n}\right)=0 \quad\left(\Delta_{A}\left(s_{n}\right)=0\right), \quad n \in \mathbf{N} .
$$

Д ок аза т е ль с т в о. Введем число $\lambda$ как действительный корень уравнения $q_{1} h_{1}^{\lambda}+\cdots+q_{n-1} h_{n-1}^{\lambda}=1$, тогда, определяя при $t>0$ функции

$$
\Delta_{M}^{\lambda}=\frac{\Delta_{M}(t)}{t^{\lambda}}, \quad \Delta_{A}^{\lambda}=\frac{\Delta_{A}(t)}{t^{\lambda}}
$$

получим

$$
\begin{aligned}
p_{1} \Delta_{M}^{\lambda}\left(h_{1} t\right)+\cdots+p_{n-1} \Delta_{M}^{\lambda}\left(h_{n-1} t\right)+\Delta_{M}^{\lambda}(t)=0, & 0<t<\delta, \\
p_{1} \Delta_{A}^{\lambda}\left(h_{1} t\right)+\cdots+p_{n-1} \Delta_{A}^{\lambda}\left(h_{n-1} t\right)+\Delta_{A}^{\lambda}(t)=0, & 0<t<\delta,
\end{aligned}
$$

где $\sum_{k=1}^{n-1} p_{k}=1$. Применим к левым частям (3.6) и (3.7) теорему о среднем, тогда

$$
\Delta_{M}^{\lambda}\left(h_{M}(t) t\right)+\Delta_{M}^{\lambda}(t)=0, \quad \Delta_{A}^{\lambda}\left(h_{A}(t) t\right)+\Delta_{A}^{\lambda}(t)=0, \quad t \in(0, \delta),
$$

где $h_{1} \leqslant h_{A}(t) \leqslant h_{n-1}$ и $h_{1} \leqslant h_{M}(t) \leqslant h_{n-1}$. Таким образом, для любого $t>0$ сушествуют две последовательности $a_{k}=a_{k}(t)$ и $b_{k}=b_{k}(t)$ такие, что

$$
h_{1}^{k} \leqslant a_{k}(t) \leqslant h_{n-1}^{k}, \quad h_{1}^{k} \leqslant b_{k}(t) \leqslant h_{n-1}^{k}
$$

и

$$
\Delta_{M}^{\lambda}\left(a_{k}(t) t\right)=(-1)^{k} \Delta_{M}^{\lambda}(t), \quad \Delta_{A}^{\lambda}\left(b_{k}(t) t\right)=(-1)^{k} \Delta_{A}^{\lambda}(t), \quad t \in(0, \delta),
$$

при $k \in \mathbf{N}$. Отсюда, в свою очередь, следует, что если $\Delta_{A}(t)$ и $\Delta_{M}(t)$ не равны тождественно нулю, то существуют последовательности $t_{k} \rightarrow 0$ и $s_{k} \rightarrow 0$ такие, что $\Delta_{M}^{\lambda}\left(t_{k}\right)=0, \Delta_{A}^{\lambda}\left(s_{k}\right)=0, k \in \mathbf{N}$. Вспоминая теперь 
определение $\Delta_{M}^{\lambda}(t)$ и $\Delta_{A}^{\lambda}(t)$ и замечая, что $\Delta_{M}(0)=\Delta_{A}(0)=0$, получим утверждение леммы.

Из леммы 3.1 следует, что либо $|f(t)|=|g(t)|$ при $|t|<\delta$, либо существует последовательность $t_{n}$, стремящаяся к нулю, такая, что $\left|f\left(t_{n}\right)\right|=\left|g\left(t_{n}\right)\right|$ при всех $n$. Во втором случае рассмотрим симметричные характеристические функции $\varphi(t)=|f(t)|^{2}$ и $\psi(t)=|g(t)|^{2}$, соответствующие функциям распределения $F(x) *(1-F(-x-0))$ и $G(x) *(1-G(-x-0))$. Согласно лемме 2.1 , эти распределения обладают моментами порядка $\alpha$. Представим число $\alpha$ в виде $2 k+\lambda$, где $0<\lambda<2$, тогда лемма 2.2 дает нам

$$
\varphi^{(l)}(0)=\psi^{(l)}(0), \quad l=0, \ldots, 2 k .
$$

Далее,

$$
\frac{\Delta_{M}(t)}{2}=\ln \varphi(t)-\ln \psi(t)=\ln \left(1+\frac{\varphi(t)-\psi(t)}{\psi(t)}\right)
$$

В силу непрерывности $\varphi(t)$ и $\psi(t)$, мы можем выбрать такое число $\delta>$ $\delta_{0}>0$, что

$$
\left|\frac{\varphi(t)-\psi(t)}{\psi(t)}\right|<\frac{1}{4}, \quad \psi(t)>\frac{2}{3}
$$

при $|t|<\delta_{0}$. Используя неравенство $\frac{2}{3}|x| \leqslant|\ln (1-x)|,|x|<\frac{1}{4}$, получим при $|t|<\delta_{0}$

$$
\left|\Delta_{M}(t)\right| \leqslant \frac{4}{3}\left|\frac{\varphi(t)-\psi(t)}{\psi(t)}\right| \leqslant 2|\varphi(t)-\psi(t)| .
$$

Разлагая функцию $\varphi(t)-\psi(t)$ в ряд Тейлора с остаточным членом в интегральной форме и учитывая (3.8), имеем

$$
\begin{aligned}
\varphi(t)-\psi(t)= & \frac{1}{(2 k-1) !} \int_{0}^{t} \varphi^{(2 k)}(t)(t-u)^{2 k-1} d u \\
& -\frac{1}{(2 k-1) !} \int_{0}^{t} \psi^{(2 k)}(t)(t-u)^{2 k-1} d u .
\end{aligned}
$$

Так как $\varphi^{(2 k)}(0)=\psi^{(2 k)}(0), \varphi^{(2 k)}(0)>\varphi^{(2 k)}(t)$ и $\psi^{(2 k)}(0)>\psi^{(2 k)}(t)$, то

$$
\begin{aligned}
|\varphi(t)-\psi(t)| \leqslant & \frac{1}{(2 k-1) !} \int_{0}^{t}\left(\varphi^{(2 k)}(0)-\varphi^{(2 k)}(t)\right)(t-u)^{2 k-1} d u \\
& +\frac{1}{(2 k-1) !} \int_{0}^{t}\left(\psi^{(2 k)}(0)-\psi^{(2 k)}(t)\right)(t-u)^{2 k-1} d u .
\end{aligned}
$$

Наша ближайшая цель - доказать, что интеграл

$$
\int_{0}^{\delta_{0}} t^{-\alpha-1}\left|\Delta_{M}(t)\right| d t
$$


сходится. Прежде всего, в силу (3.9) и (3.10) имеем

$$
\begin{aligned}
& \int_{0}^{\delta_{0}} t^{-\alpha-1}\left|\Delta_{M}(t)\right| d t \\
& \leqslant \frac{2}{(2 k-1) !} \int_{0}^{\delta_{0}} t^{\alpha-1} \int_{0}^{t}\left(\varphi^{(2 k)}(0)-\varphi^{(2 k)}(t)\right)(t-u)^{2 k-1} d u d t \\
& \quad+\frac{2}{(2 k-1) !} \int_{0}^{\delta_{0}} t^{-\alpha-1} \int_{0}^{t}\left(\psi^{(2 k)}(0)-\psi^{(2 k)}(t)\right)(t-u)^{2 k-1} d u d t
\end{aligned}
$$

Таким образом, нам достаточно доказать сходимость двух интегралов в правой части (3.12). Докажем это, например, для первого из них. Имеем

$$
\begin{gathered}
\int_{0}^{\delta_{0}} t^{-\alpha-1} \int_{0}^{t}\left(\varphi^{(2 k)}(0)-\varphi^{(2 k)}(t)\right)(t-u)^{2 k-1} d u d t \\
\times \sum_{l=0}^{2 k-1} C_{2 k-1}^{l} \int_{0}^{\delta_{0}} t^{l-\alpha-1} \int_{0}^{t} u^{2 k-1-l}\left(\varphi^{(2 k)}(0)-\varphi^{(2 k)}(u)\right) d u d t \\
=\sum_{l=0}^{2 k-1} C_{2 k-1}^{l}\left(\left.\frac{t^{l-\alpha}}{l-\alpha} \int_{0}^{t} u^{2 k-1-l}\left(\varphi^{(2 k)}(0)-\varphi^{(2 k)}(u)\right) d u\right|_{0} ^{\delta_{0}}\right. \\
\left.-\frac{1}{l-\alpha} \int_{0}^{\delta_{0}} t^{-1-\lambda}\left(\varphi^{(2 k)}(0)-\varphi^{(2 k)}(t)\right) d t\right) .
\end{gathered}
$$

Так как распределение, соответствуюшее $\varphi(t)$, обладает моментом порядка $\alpha$ и $\varphi(t)$ действительна, то по лемме 2.4

$$
\int_{0}^{t} u^{-1-\lambda}\left(\varphi^{(2 k)}(0)-\varphi^{(2 k)}(u)\right) d u<\infty
$$

По этой же причине

$$
\begin{aligned}
& \lim _{t \rightarrow 0} t^{l-\alpha} \int_{0}^{t} u^{2 k-1-l}\left(\varphi^{(2 k)}(0)-\varphi^{(2 k)}(u)\right) d u \\
& \quad \leqslant \lim _{t \rightarrow 0} \int_{0}^{t} u^{-1-\lambda}\left(\varphi^{(2 k)}(0)-\varphi^{(2 k)}(u)\right) d u=0 .
\end{aligned}
$$

Доказательство конечности интеграла в правой части (3.12), содержащего $\psi(t)$, проводится аналогично. Таким образом, интеграл (3.11) сходится, а значит,

$$
\int_{0}^{\delta} t^{-\alpha-1}\left|\Delta_{M}(t)\right| d t<\infty
$$

Определим функцию $S_{M}(u)=\Delta_{M}\left(e^{-u}\right)$ и перепишем (3.4) в виде $q_{1} S_{M}\left(u+\gamma_{1}\right)+\cdots+q_{n-1} S_{M}\left(u+\gamma_{n-1}\right)+S_{M}(u)=0, \quad u \in(-\ln \delta, \infty)$, где $\gamma_{i}=-\ln h_{i}>0, i=1, \ldots, n-1$. Не ограничивая общности рассуждений, будем считать далее $\delta=1$. 
Из (3.13) следует, что $\int_{0}^{\infty}\left|S_{M}(u)\right| e^{\alpha u} d u<\infty$, и, используя теорему 2.1 , приходим к выводу, что $S_{M}(u) \equiv 0, u>-\ln \delta$, откуда, в свою очередь, $\Delta_{M}(t) \equiv 0$, т.е. $|f(t)|^{2}=|g(t)|^{2}$ при $t \in[0, \delta)$.

Из леммы 3.1 снова выводим, что либо $\arg f(t) \equiv \arg g(t)$ на $(-\delta, \delta)$, либо существует последовательность $s_{n}$, стремящаяся к нулю и такая, что $\arg f\left(s_{n}\right)=\arg g\left(s_{n}\right)$ при всех $n$. Так как по предположению $|f(t)|$ и $|g(t)|$ не обращаются в нуль при $|t|<\delta$ и по доказанному равны там тождественно, то во втором случае

$$
f\left(s_{n}\right)=\left|f\left(s_{n}\right)\right| e^{i \arg f\left(s_{n}\right)}=\left|g\left(s_{n}\right)\right| e^{i \arg g\left(s_{n}\right)}=g\left(s_{n}\right)
$$

для достаточно больших натуральных $n$. Из леммы 2.2 следует, что $f^{(l)}(0)=g^{(l)}(0), l=0, \ldots, 2 k$.

Выберем такое $\delta>\delta_{1}>0$, что

$$
|f(t)-g(t)|<\frac{1}{4}, \quad|g(t)|>\frac{1}{2}
$$

при $|t|<\delta_{1}$. Разлагая функцию $\ln x$ в ряд Тейлора, можем написать

$$
\Delta(t)=f(t)-g(t)+\frac{1-g(t)}{g(t)}(f(t)-g(t))-\sum_{j=2}^{\infty} \frac{(g(t)-f(t))^{j}}{j g^{j}(t)} .
$$

Неравенство Коши-Буняковского дает нам

$$
\begin{aligned}
& \int_{0}^{\delta_{1}} t^{-\lambda-1}\left|\frac{1-g(t)}{g(t)} \frac{(f(t)-g(t))}{t^{2 k}}\right| d t \\
& \quad<2\left(\int_{0}^{\delta} t^{-\lambda-1}|1-g(t)|^{2} d t\right)^{1 / 2}\left(\int_{0}^{\delta} t^{-\lambda-1}\left(\frac{f(t)-g(t)}{t^{2 k}}\right)^{2} d t\right)^{1 / 2} .
\end{aligned}
$$

В силу леммы 2.3

$$
\begin{aligned}
|1-g(t)|^{2} & =(1-\operatorname{Re} g(t))^{2}+(\operatorname{Im} g(t))^{2} \leqslant(1-\operatorname{Re} g(t))^{2}+2(1-\operatorname{Re} g(t)) \\
& \leqslant 3(1-\operatorname{Re} g(t)),
\end{aligned}
$$

и поэтому по лемме 2.4 (следует учесть, что распределение $G(x)$ обладает моментом порядка $2 k+\lambda)$

$$
\int_{0}^{\delta_{1}} t^{-\lambda-1}|1-g(t)|^{2} d t \leqslant 3 \int_{0}^{\delta_{1}} t^{-\lambda-1}(1-\operatorname{Re} g(t)) d t<\infty .
$$

Далее,

$$
\begin{aligned}
f(t)-g(t)= & -\frac{1}{(2 k-1) !} \int_{0}^{t}\left(f^{(2 k)}(0)-f^{(2 k)}(t)\right)(t-u)^{2 k-1} d u \\
& +\frac{1}{(2 k-1) !} \int_{0}^{t}\left(g^{(2 k)}(0)-g^{(2 k)}(t)\right)(t-u)^{2 k-1} d u
\end{aligned}
$$


и отсюда вытекает оценка

$$
\begin{aligned}
|f(t)-g(t)|^{2} \leqslant & \frac{2 t}{(2 k-1) !} \int_{0}^{t}\left|f^{(2 k)}(0)-f^{(2 k)}(t)\right|^{2}(t-u)^{4 k-2} d u \\
& +\frac{2 t}{(2 k-) !} \int_{0}^{t}\left|g^{(2 k)}(0)-g^{(2 k)}(t)\right|^{2}(t-u)^{4 k-2} d u
\end{aligned}
$$

Опять воспользуемся первым неравенством леммы 2.3, с учетом того, что $f^{(2 k)}(t) / f^{(2 k)}(0)$ является характеристической функцией:

$$
\begin{aligned}
& \left|f^{(2 k)}(0)-f^{(2 k)}(t)\right|^{2}=\left(f^{(2 k)}(0)\right)^{2}\left|1-\frac{f^{(2 k)}(t)}{f^{(2 k)}(0)}\right|^{2} \\
& \quad=\left(f^{(2 k)}(0)\right)^{2}\left(1-\operatorname{Re} \frac{f^{(2 k)}(t)}{f^{(2 k)}(0)}\right)^{2}+\left(f^{(2 k)}(0)\right)^{2}\left(\operatorname{Im} \frac{f^{(2 k)}(t)}{f^{(2 k)}(0)}\right)^{2} \\
& \quad \leqslant 3\left(f^{(2 k)}(0)\right)^{2}\left(1-\operatorname{Re} \frac{f^{(2 k)}(t)}{f^{(2 k)}(0)}\right)
\end{aligned}
$$

Аналогично

$$
\left|g^{(2 k)}(0)-g^{(2 k)}(t)\right|^{2} \leqslant 3\left(g^{(2 k)}(0)\right)^{2}\left(1-\operatorname{Re} \frac{g^{(2 k)}(t)}{g^{(2 k)}(0)}\right) .
$$

Согласно лемме 2.4,

$$
\begin{aligned}
& \int_{0}^{\delta_{1}} t^{-1-\lambda}\left(1-\operatorname{Re} \frac{f^{(2 k)}(t)}{f^{(2 k)}(0)}\right) d t<\infty \\
& \int_{0}^{\delta_{1}} t^{-1-\lambda}\left(1-\operatorname{Re} \frac{g^{(2 k)}(t)}{g^{(2 k)}(0)}\right) d t<\infty .
\end{aligned}
$$

Объединяя последние неравенства с (3.19), (3.20) и (3.21), нетрудно вывести (аналогично тому, как это делалось при выводе (3.13)), что

$$
\int_{0}^{\delta_{1}} t^{-\lambda-1}\left|\frac{f(t)-g(t)}{t^{2 k}}\right|^{2} d t<\infty
$$

Далее, в силу (3.15)

$$
\int_{0}^{\delta_{1}} t^{-\lambda-1}\left|\frac{f(t)-g(t)}{g(t)}\right|^{j} d t \leqslant \frac{4}{2^{j-2}} \int_{0}^{\delta_{1}} t^{-\lambda-1}|f(t)-g(t)|^{2} d t
$$

и поэтому, используя (3.22), получим

$$
\left|\int_{0}^{\delta_{1}} \sum_{j=2}^{\infty} \frac{(g(t)-f(t))^{j}}{j g^{j}(t)} d t\right| \leqslant C \int_{0}^{\delta_{1}} t^{-\lambda-1}|f(t)-g(t)|^{2} d t<\infty
$$

Обозначая теперь

$$
\widetilde{\Delta}(t)=\frac{\Delta(t)}{t^{2 k}}, \quad 0<|t|<\delta
$$


имеем из последнего неравенства и неравенств (3.16)-(3.18), (3.22)

$$
\widetilde{\Delta}(t)=\frac{f(t)-g(t)}{t^{2 k}}+R(t), \quad 0<|t|<\delta_{1},
$$

где

$$
\int_{0}^{\delta_{1}} t^{-\lambda-1}|R(t)| d t<\infty
$$

В силу этого конечность интеграла

$$
\int_{0}^{\delta_{1}} t^{-\lambda-1}|\widetilde{\Delta}(a t)+\widetilde{\Delta}(b t)+\widetilde{\Delta}(-(a+b) t)| d t
$$

для любых $0<a<1,0<b<1$ эквивалентна конечности интеграла

$$
\int_{0}^{\delta_{1}} t^{-2 k-\lambda-1}\left|\frac{f(a t)-g(a t)}{a^{2 k}}+\frac{f(b t)-g(b t)}{b^{2 k}}+\frac{\overline{f((a+b) t)}-\overline{g((a+b) t)}}{(a+b)^{2 k}}\right| d t
$$

который, в свою очередь, не превосходит суммы двух

$$
\begin{gathered}
\int_{0}^{\delta_{1}} t^{-2 k-\lambda-1} \frac{1}{(2 k-1) !} \int_{0}^{t} \mid f^{(2 k)}(0)-f^{(2 k)}(a t)+f^{(2 k)}(0)-f^{(2 k)}(b t) \\
\quad+f^{(2 k)}(0)-f^{(2 k)}(-(a+b) t) \mid(t-u)^{2 k-1} d u d t \\
+\int_{0}^{\delta_{1}} t^{-2 k-\lambda-1} \frac{1}{(2 k-1) !} \int_{0}^{t} \mid g^{(2 k)}(0)-g^{(2 k)}(a t)+g^{(2 k)}(0)-g^{(2 k)}(b t) \\
+g^{(2 k)}(0)-g^{(2 k)}(-(a+b) t) \mid(t-u)^{2 k-1} d u d t .
\end{gathered}
$$

Докажем, что оба они конечны. Имеем, например, для первого

$$
\begin{array}{r}
\int_{0}^{\delta_{1}} t^{-2 k-\lambda-1} \frac{1}{(2 k-1) !} \int_{0}^{t}\left(f^{(2 k)}(0)-\operatorname{Re} f^{(2 k)}(a t)+f^{(2 k)}(0)-\operatorname{Re} f^{(2 k)}(b t)\right. \\
\left.+f^{(2 k)}(0)-\operatorname{Re} f^{(2 k)}(-(a+b) t)\right)(t-u)^{2 k-1} d u d t \\
+\int_{0}^{\delta_{1}} t^{-2 k-\lambda-1} \frac{1}{(2 k-1) !} \int_{0}^{t} \mid \operatorname{Im} f^{(2 k)}(a t)+\operatorname{Im} f^{(2 k)}(b t) \\
\quad+\operatorname{Im} f^{(2 k)}(-(a+b) t) \mid(t-u)^{2 k-1} d u d t .
\end{array}
$$

Лемма 2.3, будучи примененной $\mathrm{k}$ характеристической функции $f^{(2 k)}(t) / f^{(2 k)}(0)$, дает нам

$$
\begin{aligned}
& \left|\operatorname{Im} f^{(2 k)}(a t)+\operatorname{Im} f^{(2 k)}(b t)+\operatorname{Im} f^{(2 k)}(-(a+b) t)\right| \\
& \leqslant f^{(2 k)}(0)-\operatorname{Re} f^{(2 k)}(a t)+f^{(2 k)}(0)-\operatorname{Re} f^{(2 k)}(b t) \\
& \quad+f^{(2 k)}(0)-\operatorname{Re} f^{(2 k)}(-(a+b) t) .
\end{aligned}
$$

Теперь конечность первого интеграла в (3.23) следует из конечности интеграла

$$
\int_{0}^{\delta_{1}} t^{-2 k-\lambda-1} \int_{0}^{t}\left(f^{(2 k)}(0)-\operatorname{Re} f^{(2 k)}(t)\right)(t-u)^{2 k-1} d u d t
$$


Конечность второго интеграла в (3.23) доказывается аналогично. Таким образом, доказано, что

$$
\int_{0}^{\delta_{1}} t^{-\lambda-1}|\widetilde{\Delta}(a t)+\widetilde{\Delta}(b t)+\widetilde{\Delta}(-(a+b) t)| d t<\infty .
$$

Далее, действительнозначная функция (следует заметить, что по доказанному $\operatorname{Re} \widetilde{\Delta}(t)=0$ )

$$
N(t)=-i[\tilde{\Delta}(a t)+\tilde{\Delta}(b t)+\tilde{\Delta}(-(a+b) t)]
$$

удовлетворяет уравнению

$$
q_{1} h_{1}^{2 k} N\left(h_{1} t\right)+\cdots+q_{n-1} h_{n-1}^{2 k} N\left(h_{n-1} t\right)+N(t)=0, \quad|t|<\delta,
$$

для любых $0<a<1$ и $0<b<1$. Заметим также, что $\lambda=$ $\max \{\operatorname{Re} z: \widetilde{\tau}(z)=0\}$, где $\widetilde{\tau}(z)=1+q_{1} h_{1}^{2 k+z}+\cdots+q_{n-1} h_{n-1}^{2 k+z}$. Вводя функцию $\widetilde{S}(u)=N\left(e^{-u}\right)$, мы видим, что, во-первых, $\widetilde{S}(u)$ удовлетворяет уравнению

$$
q_{1} \widetilde{S}\left(u+2 k \gamma_{1}\right)+\cdots+q_{n-1} \widetilde{S}\left(u+2 k \gamma_{n-1}\right)+\widetilde{S}(u)=0, \quad u \in(-\ln \delta, \infty),
$$

а во-вторых (следует из (3.24)), $\int_{0}^{\infty} e^{\lambda u}|\widetilde{S}(u)| d u<\infty$. Применяя теперь теорему 2.1 , получаем, что $\widetilde{S}(u) \equiv 0$ и поэтому $N(t)=0$ при $|t|<\delta$. Таким образом,

$$
\widetilde{\Delta}(a t)+\widetilde{\Delta}(b t)+\widetilde{\Delta}(-(a+b) t)=0, \quad 0<|t|<\delta, \quad 0<a, b<1,
$$

и поэтому $\widetilde{\Delta}(t) \equiv c t, c \in \mathbf{C}$, т.е. $\Delta(t) \equiv c t^{2 k+1}$ на $(-\delta, \delta)$, но из $(3.14)$ следует, что $c=0$.

Рассмотрим теперь следующие две функции:

$$
\begin{aligned}
& f_{0}(t)=\exp \left[-|t|^{\mu}-A|t|^{\alpha}\left(1+B_{1} e^{i(t /|t|) \beta \ln |t|}+D \ln ^{m}|t|\right)\right], \\
& g_{0}(t)=\exp \left[-|t|^{\mu}-A|t|^{\alpha}\left(1-B_{2} e^{i(t /|t|) \beta \ln |t|}+D \ln ^{m}|t|\right)\right],
\end{aligned}
$$

где $z=\alpha+i \beta-$ один из корней функции $\tau(z)$ и $\mu$ выбирается следующим образом: если $\alpha \leqslant 1$, то полагаем $\mu=\alpha$; если же $\alpha \geqslant 2$, то $\mu=2$. Согласно лемме 2.6 , при подходящем выборе $A, D$ и натурального $m \leqslant 2$ эти две функции будут характеристическими для любых достаточно малых $B_{1}, B_{2}$, причем если $B_{1} \neq B_{2}$, то эти функции не равны тождественно ни на одном интервале, содержащем точку 0. Нетрудно показать также, что при любом $\varepsilon>0$ и конечном $\delta>0$

$$
\begin{aligned}
& \int_{0}^{\delta} t^{-\lambda-1+\varepsilon}\left(1-\operatorname{Re} \frac{f_{0}^{(2 k)}(t)}{f_{0}^{(2 k)}(0)}\right) d t<\infty \\
& \int_{0}^{\delta} t^{-\lambda-1+\varepsilon}\left(1-\operatorname{Re} \frac{g_{0}^{(2 k)}(t)}{g_{0}^{(2 k)}(0)}\right) d t<\infty .
\end{aligned}
$$


Поэтому, согласно лемме 1.4, соответствующие распределения имеют моменты порядка $\alpha-\varepsilon$ при $0<\varepsilon<\alpha$.

Д оказ ат ельст в о те о ремы 1.2 . Условие $M_{F} \cong M_{G}$ эквивалентно равенству

$$
F^{k_{1}}\left(b_{1} x\right) \cdots F^{k_{n}}\left(b_{n} x\right)=G^{k_{1}}\left(b_{1} x\right) \cdots G^{k_{n}}\left(b_{n} x\right), \quad x \in \mathbf{R} .
$$

Обозначим

$$
A=\sup \{x \in \mathbf{R}: F(x)=0\} .
$$

Не ограничивая общности рассуждений, предположим, что $A$ - точка непрерывности для $F(x)$, тогда из равенства (3.25) видно, что $G(x) \neq 0$ при $x>A$. Так как $A>0$ и конечно, то, беря $x=A / b_{1}$, получим снова из (3.25), что $G(A-0)=G(A+0)=0$. Таким образом, $G(x)=$ $F(x)=0, x \leqslant A$. При $x>A$ можем определить следующие функции: $\zeta(x)=-\ln F(x), \xi(x)=-\ln G(x)$, д 2 мы выбираем непрерывную ветвь логарифмической функции, принимающую вещественные значения при положительных значениях аргумента.

Из (3.25) вытекает, что

$$
k_{1} \zeta\left(b_{1} x\right)+\cdots+k_{n} \zeta\left(b_{n} x\right)=k_{1} \xi\left(b_{1} x\right)+\cdots+k_{n} \xi\left(b_{n} x\right), \quad x>b_{1}^{-1} A .
$$

Далее, обозначая $\Delta(x)=\zeta(x)-\xi(x)$, мы имеем

$$
q_{2} \Delta\left(h_{2} x\right)+\cdots+q_{n} \Delta\left(h_{n} x\right)+\Delta(x)=0, \quad x>A .
$$

Не ограничивая общности рассуждений, будем считать далее, что $A<1$. Определим при $u>0$ функцию $S(u)=\Delta\left(e^{u}\right)$ и перепишем (3.27) в виде

$$
q_{2} S\left(u+\gamma_{2}\right)+\cdots+q_{n} S\left(u+\gamma_{n}\right)+S(u)=0, \quad u \in(0, \infty),
$$

г те $\gamma_{i}=\ln h_{i}>0, i=2, \ldots, n$. Если $q_{1}+\cdots+q_{n-1} \leqslant 1$, то из теоремы 2.1 сразу получаем, что $S(u)=0$ при $u>0$ и поэтому $F(x) \equiv G(x)$ на $(1, \infty)$.

Теперь обратимся к случаю $q_{1}+\cdots+q_{n-1}>1$. Так как $F(x)$ обладает моментом порядка $\alpha_{1}>0$, то из леммы 2.8 следует

$$
-\int_{1}^{\infty} u^{\alpha_{1}-1} \ln F(u) d u<\int_{1}^{\infty} u^{\alpha_{1}-1} \frac{1-F(u)}{F(u)} d u<\infty .
$$

Отсюда и из (3.27) нетрудно вывести, что для любого $R>0$

$$
\begin{aligned}
& q_{2} h_{2}^{\alpha_{1}} \int_{h_{2}}^{h_{2} R} u^{\alpha_{1}-1} \xi(u) d u+\cdots+q_{n} h_{n}^{\alpha_{1}} \int_{h_{n}}^{h_{n} R} u^{\alpha_{1}-1} \xi(u) d u \\
& \quad+\int_{1}^{R} u^{\alpha_{1}-1} \xi(u) d u<\infty .
\end{aligned}
$$

Отсюда, в свою очередь, следует, что $\int_{1}^{\infty} u^{\alpha_{1}-1} \xi(u) d u<\infty$. Таким образом,

$$
\int_{1}^{\infty} u^{\alpha_{1}-1}|\Delta(u)| d u<\int_{1}^{\infty} u^{\alpha_{1}-1} \xi(u) d u+\int_{1}^{\infty} u^{\alpha_{1}-1} \zeta(u) d u<\infty
$$


и поэтому $\int_{0}^{\infty}|S(u)| e^{\alpha_{1} u} d u<\infty$. Опять, использую теорему 2.1 , получаем $S(u)=0, u>0$.

Докажем теперь, что $\Delta(x)=0$ при $x \in(A, 1)$. Рассмотрим основное уравнение (3.27), которое имеет место при $x>A$. Беря $x$ из $\left[h_{2}^{-1}, \infty\right) \cap$ $(A, 1)$, получим, что $\Delta(x)=0$. Повторяя этот процесс $k$ раз, приходим к выводу, что $\Delta(x)=0, x \in\left[h_{2}^{-k}, \infty\right) \cap(A, 1)$. Осталось вспомнить, что $h_{2}>1$. Рассмотрим теперь при $x>0$ две функции

$$
\begin{aligned}
& F_{0}(x)=\exp \left\{-x^{-\alpha_{1}}\left(1+C_{1} \cos (\beta \ln x)\right)\right\}, \\
& G_{0}(x)=\exp \left\{-x^{-\alpha_{1}}\left(1+C_{2} \cos (\beta \ln x)\right)\right\},
\end{aligned}
$$

где $\alpha_{1}+i \beta-$ один из корней функции $\tau_{1}(z)$. Согласно лемме 2.7 , эти две функции являются функциями распределения на $(0, \infty)$ при $\max \left(\left|C_{1}\right|,\left|C_{2}\right|\right)<1+|\beta| / \alpha$, причем они различны, если $C_{1} \neq C_{2}$. Из теоремы 2.1 нетрудно вывести, что $F_{0}(x)$ и $G_{0}(x)$ удовлетворяют уравнению (3.27), т.е. распределения статистики $M$, соответствующие им, совпадают. Далее, так как функции $x^{\alpha_{1}-\delta-1}\left(1-F_{0}(x)\right)$ и $x^{\alpha_{1}-\delta-1}\left(1-G_{0}(x)\right)$ интегрируемы на $(0, \infty)$ при $\alpha_{1}>\delta>0$, то, согласно следствию 2.2 , $F_{0}(x)$ и $G_{0}(x)$ обладают моментами порядка $\alpha_{1}-\delta$.

\section{СПИСОК ЛИТЕРАТУРЫ}

1. Беломестный Д. В. К вопросу о восстановлении распределения слагаемых по распределению суммы. - Теория вероятн. и ее примен., 2001, т. 46, № 2, с. 366-370.

2. Крейн М.Г. О проблеме продолжения эрмитово-положительных непрерывных функций. - Докл. АН СССР, 1940, т. 26, № 1, с. 17-21.

3. Johnson $N L$., Kotz $S$. Characterization of an absolutely continuous distribution by the distribution of any order statistics. - Indian J. Math., 1990, v. 32, № 2, p. 193194.

4. Kawata T. Fourier Analysis in Probability Theory. New York-London: Academic Press, 1972, $668 \mathrm{p}$.

5. Линник Ю.В. K вопросу о нахождении генерального распределения по распределению статистики. - Теория вероятн. и ее примен, 1956, т. 1, №4, c. $466-478$.

6. Линник Ю. В. Линейные формы и статистические критерии. I, II. - Укр. матем. журн., 1953, т. 5, № 2, № 3, с. 207-243; 247-290.

7. Петров В. В. Предельные теоремы для сумм независимых случайных величин. M.: Наука, 1987, 317 с.

8. Прохоров А.В., Ушаков Н.Г. О задаче восстановления распределения слагаемых по распределению суммы. - Теория вероятн. и ее примен., 2001, т. 46, № 3, с. 449462 .

9. Ushakov N. G. Selected Topics in Characteristic Functions. Utrecht: VSP, 1999, 355 p.

10. Феллер В. Введение в теорию вероятностей и ее приложения. Т. 2. М.: Мир, 1984, $751 \mathrm{c}$.

11. Belomestnyi $D . V$. On the problem of characterizing the distribution of random variables by the distribution of their sum. - J. Math. Sci. (New York), 2002, v. 111, № 3, p. 3498-3504. 